\title{
Kinetic Factors in the Tooth-Plaque-Saliva Model Systems
}

\author{
W. I. HIGUCHI \\ College of Pharmacy, University of Michigan, Ann Arbor, Michigan 48104, USA
}

During the last decade the physical model approach has been leading to a systematic, selfconsistent, mechanistic understanding of the demineralization kinetics of dental enamel reactions in acidic media. ${ }^{1,2}$ In recent apatite dissolution studies it has aided greatly in defining the exact roles of solution fluoride and strontium and in elucidating the fluoride-strontium-phosphate synergism in retarding acid demineralization. ${ }^{3-\bar{a}}$

The proposed model for the tooth-plaquesaliva system is intended to correlate, within the plysicochemical framework, the various kinetic factors of the tooth, plaque, and saliva evidenced to be directly or indirectly related to dental caries. In the light of appropriate experimental designs, investigations based on such models of ten may provide quantitative interrelationships that would not be possible intuitively; this has already been demonstrated in a preliminary study. 6

\section{The General Model}

This model may describe the dynamic processes in dental caries. The enamel subsurface demineralization is shown in the illustration as the consequence of events taking place in the saliva, dental plaque, and tooth surface regions. The essence of this model is derived from the "acid theory" and white spot formation in caries etiology. Thus, acids may be produced from bacterial metabolism in the cariogenic plaque layer, which is constantly bathed in the saliva containing the necessary substrate (s). Tooth mineral dissolution then is induced by these acids. The illustration shows that during dissolution the model requires that diffusion of various species takes place in the apparent intact tooth surface enamel layer as well as in the plaque. While calcium and the phosphate species diffuse away from the subsurface region into saliva, saliva substrate is simulta- neously diffusing into the plaque and being used by the bacteria. The acids generated in the plaque $\left(\mathrm{H}, \mathrm{HL}\right.$, and $\left.\mathrm{H}_{2} \mathrm{C}\right)$ diffuse both into the subsurface region and toward the saliva.

In this tooth-plaque-saliva system, the following kinetic factors that contribute to the demineralization process can be identified: (1) the concentration gradients and transport rates of various species in the plaque;

(2) the nature of the plaque bacteria, bacterial acid, and acid production rates; (3) the thickness (or amount) of the cariogenic plaque; (1) the transport rates of the various species in the intact surface enamel layer; (5) enamel apatite-ambient solution interfacial conditions in the subsurface demineralizing region; and (6) the saliva calcium and phosphate levels, buffer capacity and $\mathrm{pH}$, and substrate levels. Considerable evidence has accumulated indicating, directly or indirectly, that combinations of these factors are involved in forming the necessary conditions for caries formation. One factor may not merit greater consideration than another without proper evaluation of its role.

In his presentation Dr. Brown has discussed the possible importance of the thermodynamic (equilibrium solubility) factors in the caries process. It would be shortsighted, however, if the unusual and slow crystalsolution kinetics generally observed with synthetic apatite and dental enamel is disregarded completely as being unimportant to dental caries. Our own thoughts (at this time) are that crystal-solution kinetics may be as important as thermodynamics in the initiation of dental caries and, therefore, should be given appropriate consideration.

\section{Potential Usefulness of the Model}

The physical model approach to the caries problem is unique in that it correlates, in perspective, the various factors identified in 


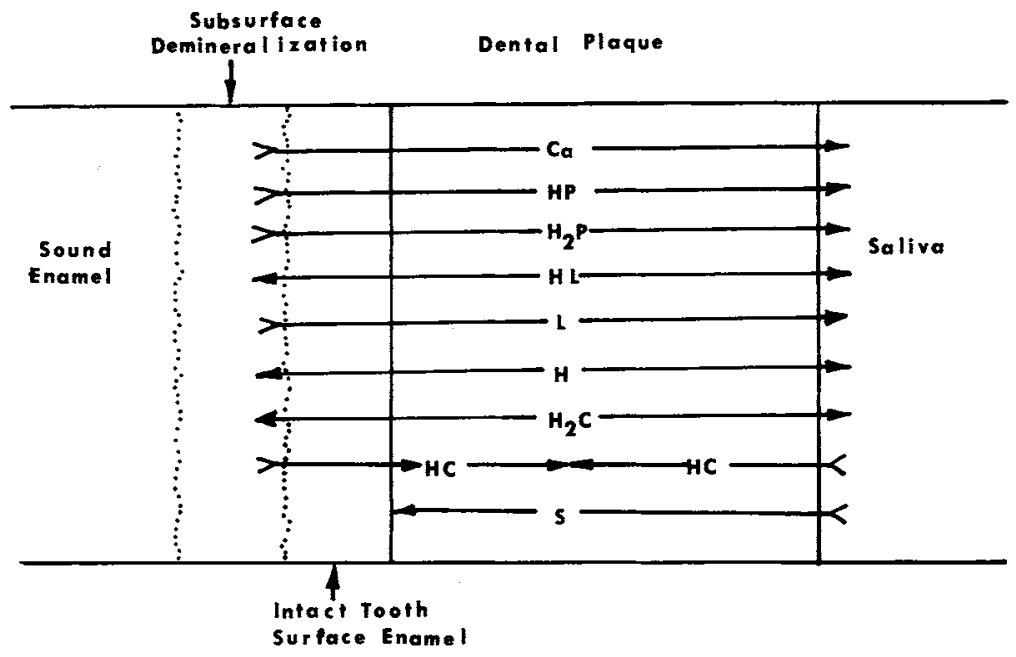

FIG 1.-Diagram for enamel subsurface demineralization in a tooth-plaquesaliva system. $\mathrm{Ca}=\mathrm{Ca}^{++} ; \mathrm{HP}=\mathrm{HPO}_{\overline{7}}^{-} \mathrm{H}_{2} \mathrm{P}=\mathrm{H}_{2} \mathrm{PO}_{\overline{1}}^{-} ; \mathrm{H}=\mathrm{H}^{+} ; \mathrm{H}_{2} \mathrm{C}=\mathrm{H}_{2} \mathrm{CO}_{3}$; $\mathrm{HC}=\mathrm{HCO}_{3} ; \mathrm{HL}=$ bacteria-produced acid molecules; $\mathrm{L}=$ anions of $\mathrm{HL} ; \mathrm{S}=$ substrate (carbohydrate).

many independent investigations. These relationships and appropriately designed experiments may provide the basis for a "systems approach" to caries research.

Based on the dynamic processes in the tooth-plaque-saliva system and the other physicochemical factors, quantitative integral mathematical relationships can be derived in terms of tooth subsurface demineralization as a function of various factors relating to apatite dissolution kinetics, plaque (or bacterial) cariogenicity, and saliva conditions.

Examples of phenomena related to caries that may be mechanistically investigated and quantitated by the proposed approach include (1) subsurface dissolution itself; (2) enzymatic glycolysis of carbohydrates by cariogenic bacteria in the plaque; (3) the actions of agents that may act primarily in the plaque (antibacterials); (4) the actions of agents that may increase the resistance of the enamel to demineralization (fluoride, trace elements, and other agents); and (5) saliva composition and the influences of the variations in the saliva composition with time (cycling effects).

\section{Recommendations}

General.-It has been evident from this workshop that many of the "pieces in the puzzle" have been found and more undoubtedly need to be found before the picture can be completed. I believe, however, that enough is now known about the caries pro. cess so that physical models for the dynamic tooth-plaque-saliva system can be helpful in putting the existing pieces together.

The investigator with physicochemical (or perhaps biochemical engineering) inclinations may make a significant contribution by developing and using the "systems approach" to the mechanistic understanding of dental caries and rational approaches to the elimination of this disease. Success, to a large extent, will depend on the investigator's ability to communicate effectively with microbiologists, biochemists, and clinicians engaged in caries research, as well as on the investigator's proficiency in mathematics.

SPECIFIC.- The relationships between enamel apatite crystal dissolution kinetics in the "white spot" zone and the time changes in the morphology must be established. In this connection, crystal dissolution studies under partial saturation conditions will probably yield important results.

The influence of trace elements and other agents (including crystal poisons) on the apparent solubility and the kinetics of dissolution of hydroxyapatite should be investigated from a mechanistic standpoint.

The permeability of enamel (especially the sound outer layer with and without the pellicle) to ionic and nonionic substances 
must be determined. In this connection, the possible permselective nature of the enamel should be investigated.

The permeability of the plaque to nonionic and ionic compounds of interest in the toothplaque-saliva system must be determined.

\section{References}

1. Higuchi, W.I.; Gray, J.A.; Hefferren, J.J.; and Patel, P.R.: Mechanisms of Enamel Dissolution in Acid Buffers, $J$ Dent Res 44: 330$341,1965$.

2. Higuchi, W.I.; Mir, N.A.; Patel, P.R.; Becker, J.W.; and Hefrerren, J.J.: Quantitation of Enamel Demineralization Mechanisms: III. A Critical Examination of the Hydroxyapatite, $J$ Dent Res 48: 396-409, 1969.
3. Mir, N.A.; Higuchi, W.I.; and HefFerRen, J.J.: The Mechanism of Action of Solution Fluoride Upon the Demineralization Rate of Human Enamel, Arch Oral Biol 14: 901-920, 1969.

4. Dedhiya, M.G.; Young, F.; and Higuchi, W.I.: Mechanism for the Retardation of the Acid Dissolution Rate of Hydroxyapatite by Strontium, J Dent Res 52: 1097-1109, 1973.

5. DedhiYa, M.G.; Young, F.; and Higuchi, W.I.: Mechanism of Hydroxyapatite Dissolution. The Synergistic Effects of Solution Fluoride, Strontium, and Phosphate, $J$ Phys Chem, in press.

6. Higuchi, W.I.; Young, F.; Lastra, J.L.; and Koulourides, T.: Physical Model for Plaque Action in the Tooth-Plaque-Saliva System, $J$ Dent Res 49: 47-60, 1970. 